\title{
A Study of Vitamin-C Level as Oxidative Stress Marker In chronic Renal Failure Patients
}

\author{
Dr. Praveen Kumar G $S^{1}$, Miss. Meghalatha T $S^{2} \&$ Dr. Arup Kumar Banerjee \\ ${ }^{1}$ Associate professor $\&$ Tutor, Department of Biochemistry, KarpagaVinayaga Medical College \& Research \\ Center, Kancheepuram, Tamil Nadu. \\ ${ }^{3}$ Assistant professor, Department of Biochemistry, PES Medical College, Kuppam, Andhra Pradesh
}

\begin{abstract}
:
Aim: The aim of this studywas to measure the Vitamin-C levels in chronic kidney disease patients. Chronic renal failure is a clinical syndrome resulting in the progressive loss of renal function. The symptoms of chronic renal failure result not only from simple excretory failure but also from the onset of regulating failure, the kidney's failure to regulate certain substances, such as sodium \& water. According to National Kidney Foundation- Kidney Dialysis Outcomes Quality Initiative (NKF-KDOQI) guidelines CRF is classified into 5 stages. An increased oxidative stress and inflammation are both common features of end stage renal disease.

Methods: Here, we determined the vitamin-C levels in patients with chronic kidney disease and the antioxidant status of vitamin-C in these patients.

Results: The mean plasma Vitamin - $C$ concentration has found to be decreased in CKD patients when compared with the controls.

Conclusion: Vitamin $C$ improves endothelial function in the resistance vasculature of patients with chronic renal failure, and this effect was abolished by an inhibitor of nitric oxide (NO) synthesis.There is insufficient evidence to recommend the use of vitamin C (ascorbate) in the management of patients with CKD.
\end{abstract}

Keywords: Oxidative stress, Antioxidant, Vitamin-C, Chronic renal failure (CRF).

\section{Chronic renal failure (CRF) \& Oxidative stress}

\section{Introduction}

Chronic renal failure (CRF) is a clinical syndrome resulting the progressive loss of renal function.According to National Kidney Foundation- Kidney Dialysis Outcomes Quality Initiative (NKFKDOQI) guidelines CRF is classified into 5 stages. An increased oxidative stress and inflammation are both common features of end stage renal disease. It has been speculated that there may be an association between oxidative stress and endothelial dysfunction contributing to an increased risk for cardiovascular disease. Here, we determine the vitamin-C levels in patients with chronic kidney disease and the antioxidant status of vitamin$\mathrm{C}$ in these patients. An antioxidant is a molecule capable of inhibiting the oxidation of other molecules. Oxidation is a chemical reaction that transfers electrons from a substance to an oxidizing agent. Oxidation reactions can produce free radicals. In turn, these radicals can start chain reactions that damage cells. Antioxidants terminate these chain reactions by removing free radical intermediates, and inhibit other oxidation reactions. Fruits and vegetables are good sources of antioxidants.

\section{Oxidative Stress \& Vitamin-C}

Oxidative stress is thought to contribute to the development of a wide range of diseases. However, it is unknown whether oxidative stress is the cause or the consequence of disease.Ascorbic acid or "vitamin $\mathrm{C}$ " is a monosaccharide, oxidation-reduction (redox) catalyst found in both animals and plants. As one of the enzymes needed to make ascorbic acid has been lost by mutation during primate evolution, humans must obtain it from the diet; it is therefore a vitamin. 


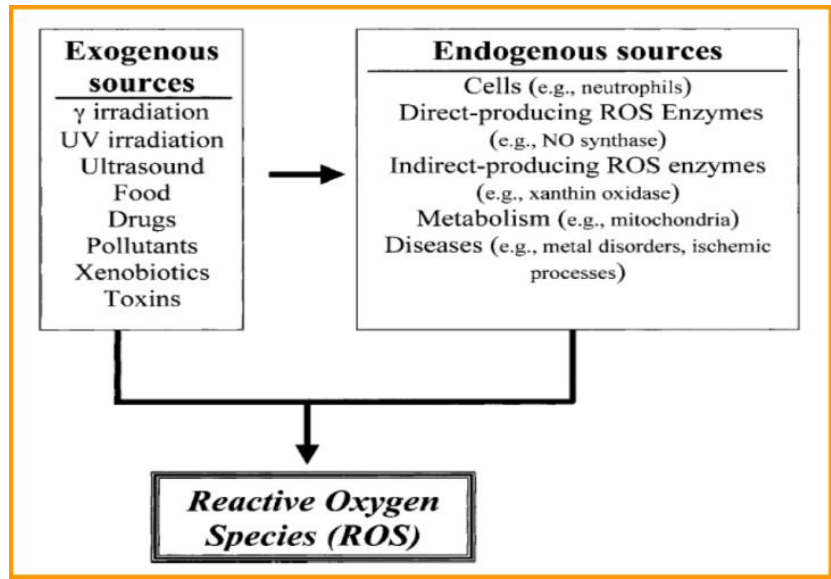

\section{Antioxidant role of vitamin-C}

Can react with many reactive oxygen species (ROS) and reactive nitrogen species (RNS).Can regenerate vitamin $\mathrm{E}$

Aim

To measure the Vitamin-C levels in chronic kidney disease patients.

Subjects

\section{Methods \& Materials}

Chronic kidney disease patients were selected on the basis of serum creatinine levels. Serum creatinine $>\mathbf{2 . 5} \mathbf{m g} / \mathbf{d l}$ values were considered to be having chronic kidney disease. Both male and females patients with age ranging from 20-70yrs are taken in this study.

Study design: -

The conducted study was a Case Control comparative study.

\section{Target population:-}

Target population was patients admitted in the Nephrology department of PES Hospital, Kuppam.

Sample size:

In this study we have measure the Vitamin - C levels in 30 chronic kidney disease patients and in 30 controls.

Exclusion criteria:

Patients with cardiac disease, pregnant women and those who required urgent dialysis are excluded from this study.

Inclusion criteria:

Patients with all stages of chronic kidney disease of any causes admitted in the Nephrology department of PESIMSR, Kuppam are included in the study.

Methodology and data collection

1. Collection of serum samples

- Serum samples were collected from the patients admitted in Nephrology department of PES Hospital.

- $5 \mathrm{ml}$ of venous blood was collected from the patients.

- $\quad$ All assays were performed within $48 \mathrm{hrs}$ of separation of serum.

- $\quad$ The collected samples were stored air tight at $2^{0} \mathrm{C}-8^{0} \mathrm{C}$ in the refrigerator.

\section{Biochemical analysis}

The serum vitamin - C levels are estimated by EVELYN \& MELLOY method manually by using colorimeter. The present study comprises of 30 clinically diagnosed cases of chronic kidney disease. The age group ranges from 20-70 years, out of these, 19 patients were males and 11 patients were females. Statistical data analyses were performed using "Excel".

\section{Results}

The mean plasma Vitamin - C concentration has found to be decreased in CKD patients when compared with the controls. 


\begin{tabular}{|c|c|c|c|c|c|c|}
\hline & & $\mathrm{N}$ & Mini. & Maxi. & Mean & SD \\
\hline CKD & Vit C $\quad(\mathrm{mg} / \mathrm{d}$ & 30 & 0.03 & 0.33 & 0.181 & 0.07089 \\
\hline Controls & Vit C $(\mathrm{mg} / \mathrm{dl})$ & 30 & 0.40 & 1.99 & 1.0177 & 0.34697 \\
\hline
\end{tabular}

Diagram.1. vitamin-C levels in controls and chronic renal failure patients

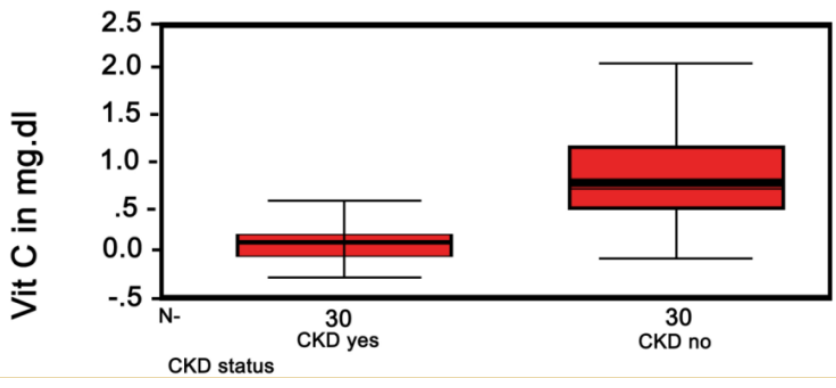

IV. Conclusion

Vitamin- $\mathrm{C}$ is excreted by the kidney due to its water solubility. Renal dysfunction may be associated with a decrease in plasma Vitamin- $\mathrm{C}$ levels.Moreover, the decreased vitamin- $\mathrm{C}$ may cause endothelial dysfunction.There is insufficient evidence to recommend the use of vitamin $\mathrm{C}$ (ascorbate) in the management of patients with CKD.

\section{Discussion}

Vitamin C improves endothelial function in the resistance vasculature of patients with chronic renal failure, and this effect was abolished by an inhibitor of nitric oxide (NO) synthesis. Uremic patients have an increased incidence of endothelial dysfunction and oxidative stress that can contribute to cardiovascular (CV) events. Intake of greater than 100-200 mg/day of Vitamin- C should be avoided in CKD to avoid oxalosisbecause vitamin $\mathrm{C}$ is excreted by the kidney -which is the site of accumulation of oxalate, which is the metabolic by-product of ascorbic acid. Balcke et al, working with long-term dialysis patients, demonstrated that an increased level of oxalate concentration indicates a most important risk factor for calcium oxalate deposits in uremic patients.

\section{References}

[1]. Clinical chemistry (Theory, Analysis, Correlation) by Lawrence A Kaplan et.al , $4^{\text {th }}$ Edition, Page: 486

[2]. Harrison's Internal Medicine. Vol.2, $17^{\text {th }}$ Edition, Page:1762.

[3]. Tietz text book of clinical chemistry and molecular diagnosis. $4^{\text {th }}$ Edition, Page: 1694

[4]. Francesco Localelliet.al., Oxidative stress in end stage renal disease: an emerging threat to patient outcome.,NDT: 2003 Jul.;18(7):1272-80.

[5]. Nobuyuki Tkahashiet.al.,Decreased plasma levels of Vitamin- C in Chronic Kidney Disease: comparison between diabetic \& non diabetic patients. NDT:2010 Sept-3

[6]. Debra Blair et.al.Vitamin- C supplementation \& chronic kidney disease., Renal nutrition update, June-15, 2009.

[7]. Sies H (1997)."Oxidative stress: oxidants and antioxidants". ExpPhysiol 82 (2): 291-5.

[8]. Smirnoff N (2001)."L-ascorbic acid biosynthesis". VitamHorm 61: $241-66$.

[9]. Linster CL, Van Schaftingen E (2007). "Vitamin C. Biosynthesis, recycling and degradation in mammals". FEBS J. 274 (1): 1-22.

[10]. Jenny M Cross, Raymond J MacAllister and Robin G Woolfson., Vitamin C in renal failure-Pro- or antioxidant?.,Kidney International (2003) 64, 1531-1532;

[11]. Ghiadoni L, Cupisti A, Huang Y, Mattei P, Cardinal H, Favilla S, Rindi P, Barsotti G, Taddei S, Salvetti A. J Nephrol., Endothelial dysfunction and oxidative stress in chronic renal failure.2004 Jul-Aug;17 (4):512-9 